\title{
IFN-Alpha-Induced Cortical and Subcortical Glutamate Changes Assessed by Magnetic Resonance Spectroscopy
}

\author{
Ebrahim Haroon', Bobbi J Woolwine', Xiangchuan Chen ${ }^{2}$, Thaddeus W Pace ${ }^{3}$, Samir Parekh ${ }^{4}$, \\ James R Spivey ${ }^{4}$, Xiaoping $\mathbf{P} \mathbf{H u}^{2}$ and Andrew H Miller*, I \\ 'Department of Psychiatry and Behavioral Sciences, Emory University School of Medicine, Atlanta, GA, USA; ${ }^{2}$ Coulter BME Biomedical Imaging \\ Technology Center, Emory University School of Medicine, Atlanta, GA, USA; ${ }^{3}$ Department of Psychiatry, College of Medicine, University of Arizona, \\ Tucson, AZ, USA; ${ }^{4}$ Department of Medicine, Division of Gastroenterology, Emory University School of Medicine, Atlanta, GA, USA
}

\begin{abstract}
Cytokine effects on behavior may be related to alterations in glutamate metabolism. We therefore measured glutamate concentrations in brain regions shown to be affected by inflammatory stimuli including the cytokine interferon (IFN)-alpha. IFN-alpha is known to alter neural activity in the dorsal anterior cingulate cortex (dACC) and basal ganglia in association with symptoms of depression and increases in peripheral cytokines including the tumor necrosis factor (TNF) and its soluble receptor. Single-voxel magnetic resonance spectroscopy (MRS) was employed to measure glutamate concentrations normalized to creatine $(\mathrm{Glu} / \mathrm{Cr}$ ) in $\mathrm{dACC}$ and basal ganglia of 31 patients with hepatitis $C$ before and after $\sim 1$ month of either no treatment $(n=14)$ or treatment with IFN-alpha $(n=17)$. Depressive symptoms were measured at each visit using the Inventory of Depressive Symptoms-Clinician Rating (IDS-C) and the Multidimensional Fatigue Inventory. IFN-alpha was associated with a significant increase in Glu/Cr in dACC and left basal ganglia. Increases in dACC Glu/Cr were positively correlated with scores on the IDS-C in the group as a whole, but not in either group alone. Glu/Cr increases in left basal ganglia were correlated with decreased motivation in the group as a whole and in IFN-alpha-treated subjects alone. No Glu/Cr changes were found in the right basal ganglia, and no significant correlations were found between $\mathrm{Glu} / \mathrm{Cr}$ and the inflammatory markers. IFN-alphainduced increases in glutamate in $\mathrm{AACC}$ and basal ganglia are consistent with MRS findings in bipolar depression and suggest that inflammatory cytokines may contribute to glutamate alterations in patients with mood disorders and increased inflammation.

Neuropsychopharmacology (2014) 39, 1777-1785; doi:10.1038/npp.2014.25; published online 26 February 2014
\end{abstract}

Keywords: glutamate; cytokines; interferon-alpha; anterior cingulated; basal ganglia; anhedonia

\section{INTRODUCTION}

Evidence suggests that inflammatory cytokines target cortical and subcortical brain regions that mediate effects of inflammation on behavior. Neuroimaging studies have shown that administration of cytokines or cytokine inducers can alter neural activity in basal ganglia and anterior cingulate cortex (dACC) leading to behavioral changes. For example, using functional magnetic resonance imaging (fMRI), the inflammatory cytokine interferon (IFN)-alpha was found to decrease neural activation in ventral striatum during a reward-processing task, which correlated with reduced motivation (Capuron et al, 2012). Similar results were found following administration of endotoxin to healthy volunteers, who also exhibited reduced ventral

*Correspondence: Dr AH Miller, Department of Psychiatry and Behavioral Sciences, Emory University School of Medicine, Winship Cancer Institute, I365-B Clifton Rd., Room B5 I 0 I, Atlanta, GA 30322, USA, Tel: 404727 8260, Fax: 404778 3965,

E-mail: amill02@emory.edu

Received 20 August 2013; revised 19 January 2014; accepted 21 January 2014; accepted article preview online 31 January 2014 striatal activation during a monetary reward task that correlated with depressed mood (Eisenberger et al, 2010). In addition, healthy volunteers exposed to typhoid vaccination (compared with placebo) showed altered activation in the substantia nigra that was associated with reduced psychomotor speed and increased peripheral blood interleukin-6 (Brydon et al, 2008).

Cytokine effects on the anterior cingulate cortex (ACC) have also been found in the context of exposure to multiple inflammatory stimuli. Increased activation of dorsal ACC (deACC) (Broadman's area (BA) 24) in response to a 'location-detection' visuospatial challenge paradigm was seen during fMRI in hepatitis C patients treated with IFN-alpha compared with controls (Capuron et al, 2005). In addition, administration of typhoid vaccination was associated with increased dACC activation compared with placebo using a Stroop task in healthy volunteers (Harrison et al, 2009). Increased secretion of soluble tumor necrosis factor receptor 2 (sTNFR2) during psychosocial stress has also been found to correlate with greater dACC activation in response to social isolation using a cyberball-exclusion paradigm during fMRI (Slavich et al, 2010). Thus, the basal ganglia and AACC appear to represent important targets of 
immune activation in multiple paradigms using several distinct immune stimuli, ultimately leading to behavioral changes.

One mechanism that may underlie these cytokine effects on neural activity is altered glutamate metabolism. Increased glutamate neurotransmission is believed to contribute to behavioral alterations in mood disorders, and inflammatory cytokines have been shown to increase synaptic and extrasynaptic concentrations of glutamate by decreasing glutamate transporters and increasing glutamate release from relevant glial elements including astrocytes (Tilleux and Hermans, 2007; Ida et al, 2008; Sanacora et al, 2012). Of note, IFN-alpha-induced neurotoxicity in the human immunodeficiency virus (HIV) encephalitis mouse model has also been shown to be mediated by increased $\mathrm{N}$-methyl-D-aspartate (NMDA) signaling, presumably secondary to increased glutamate activation of NMDA receptors (Sas et al, 2009). Moreover, patients with bipolar depression have been reliably found to exhibit increased glutamate in the ACC using magnetic resonance spectroscopy (MRS) and also exhibit postmortem evidence of neuroinflammation including microglial activation (Yuksel and Ongur, 2010; Stertz et al, 2013). Finally, blockade of NMDA receptors using ketamine has been shown to reverse behavioral changes induced by activation of inflammatory pathways by lipopolysaccharide (Walker et al, 2013).

To further examine the impact of inflammatory cytokines on glutamate metabolism in the brain, we conducted MRS before and after the 4 th dose of IFN-alpha in patients with hepatitis $\mathrm{C}$ virus $(\mathrm{HCV})$. We hypothesized that IFN-alpha would increase glutamate concentrations in basal ganglia and dACC as measured by single-voxel proton MRS. Singlevoxel MRS allows detection of a variety of neurometabolite markers, typically in concentrations of $0.5-10 \mathrm{mM}$ within cells or in extracellular spaces, including glutamate (van der Graaf, 2010). MRS glutamate assessments were correlated with changes in behavior as well as changes in the plasma concentration of TNF and sTNFR2, both of which have been associated with IFN-alpha-induced behavioral changes including depression and fatigue (Raison et al, 2009).

\section{MATERIALS AND METHODS}

Thirty-one HCV-positive patients (20 females and 11 males) were enrolled in the study. All subjects were serum positive for HCV-RNA by reverse transcription-PCR. Exclusion criteria were: liver failure; decompensated or advanced cirrhosis (Child-Pugh Score $>6$ ); liver disease from any cause other than HCV; HIV infection; unstable cardiovascular, endocrinologic, hematologic, renal or neurological disease (as determined by laboratory testing and physical examination); current major depression, substance abuse and/or dependence within the past year; or lifetime history of schizophrenia or bipolar disorder as determined by Structured Clinical Interview for Diagnostic and Statistical Manual of Mental Disorders-Fourth Edition (SCID IV) (First et al, 2002). Anxiety disorders were not exclusionary. Patients were required to be antidepressant-free for at least 1 year and off all other psychotropic medications for at least 1 month before MRS scanning. Of note, one patient was allowed to participate after taking a sedative/hypnotic the night before the scan. In addition, patients had to be free of contraindications to MRI including metallic implants and claustrophobia.

\section{Study Design}

A prospective, longitudinal design was employed. Patients in the IFN-alpha treatment group $(n=17)$ underwent MRS scans, psychiatric assessments, and blood sampling within 7 days before starting IFN-alpha administration (baseline or visit 1) and within 4-6 days following their fourth IFN-alpha injection (visit 2). A HCV-control group $(n=14)$ awaiting IFN-alpha, underwent MRS scans, psychiatric ratings, and blood draws at baseline (visit 1) and $\sim 1$ month later (visit 2), similar to the IFN-alpha treatment group. Mean time from visit 1 to visit 2 was 37.1 SD 12.0 days for IFNalpha-treated subjects and 31.6 SD 6.0 days for controls $(t=-1.55, \mathrm{df}=29, p=0.13)$. Assignment to the treatment $v s$ control group and type of IFN-alpha was determined by patients and their physicians and was not controlled by the study protocol. Hepatitis $C$ patients in the IFN-alpha group were treated with either pegylated IFN-alfa-2b (Pegintron, Merck $1.5 \mathrm{mcg} / \mathrm{kg} /$ week SC) $(n=1)$ or pegylated IFN-alfa-2a (Pegasys, Roche $184 \mathrm{mcg} /$ week/kg SC) $(n=16)$ plus ribavirin $(800-1200 \mathrm{mg} / \mathrm{d})(n=17)$ in the presence or absence of telaprevir $(750 \mathrm{mg}$ p.o. t.i.d.) $(n=10)$ or boceprevir $(800 \mathrm{mg}$ p.o. t.i.d. $)(n=1)$ as determined by their treating physician. Non-fasting blood samples were collected between 1 and $2 \mathrm{pm}$ to reduce circadian variation in inflammatory mediators, although previous studies by our group have not found circadian variations in TNF or sTNFR2 in this patient population (unpublished observations). All study procedures were approved a priori by the Emory University institutional review board, and all subjects provided written informed consent.

\section{Behavioral Assessments}

Depression was evaluated by the Inventory for Depressive Symptomatology-Clinician Rating (IDS-C); a 30-item scale that provides valid and reliable ratings of severity of depressive symptoms, including depressed mood, guilt, suicidal ideation, sleep, appetite, energy, libido, and concentration (Trivedi et al, 2004). Clinician ratings were not blinded to group assignment, as it is difficult to mask the profound behavioral effects of IFN-alpha.

To evaluate severity of fatigue, subjects completed the self-rated 20-item Multidimensional Fatigue Inventory (MFI-20) (Smets et al, 1995). The MFI assesses five dimensions of fatigue known to be common among medically ill patients including general fatigue, physical fatigue, mental fatigue, reduced activity, and reduced motivation (Stein et al, 2004).

\section{Plasma Inflammatory Cytokines}

Plasma was isolated from centrifuged whole blood collected in chilled EDTA tubes. Samples were stored at $-80^{\circ} \mathrm{C}$ for the batched assay. Plasma TNF and sTNFR2 were measured by quantitative enzyme-linked immunosorbent assays (R \& D Systems, Minneapolis, MN). Assays were performed according to manufacturer's specifications and were run in 
duplicate. Inter- and intra-assay variability were reliably $<12 \%$ for TNF and $<10 \%$ for sTNFR2. Lower limits of detection for TNF and sTNFR2 were $0.19 \mathrm{pg} / \mathrm{ml}$ and $2.3 \mathrm{ng} / \mathrm{ml}$, respectively. All biological samples were analyzed by research staff blinded to the clinical status of study participants, and no samples were below the limit of detection.

\section{Neuroimaging Protocol}

MRS acquisition and processing. The scanning protocol and post-processing methodology have been previously reported (Ajilore et al, 2005; Ajilore et al, 2007; Haroon et al, 2009). Briefly, all images were acquired using a Siemens 3 Tesla Magnetom Trio System 3.0 (Siemens Medical Solutions, Malvern, PA) equipped with $40 \mathrm{mT} / \mathrm{m}$ capable Sonata gradient set with a maximum rise time of $200 \mu$ s. Subjects were positioned supine with their whole body inside the scanner bore. A transverse electromagnetic head coil was used for excitation and detection. For image guidance and MRS volume of interest, anatomical T1 images were obtained using 3D Magnetization Prepared RApid Gradient Echo (MPRAGE) with settings: TR $=2300$ $\mathrm{ms}, \mathrm{TE}=3.02 \mathrm{~ms}, \mathrm{TI}=1100 \mathrm{~ms}$, flip angle $=8^{\circ}$ and voxel size $1 \times 1 \times 1 \mathrm{~mm}^{3}$. Two voxels each of $17 \times 30 \times 17 \mathrm{~mm}^{3}$ located on the right and left basal ganglia and one voxel sized $20 \times 30 \times 10 \mathrm{~mm}^{3}$ located on the dACC (BA24) were used to obtain single-voxel $\mathrm{H}^{1}$-MRS (Figure 1). The spatial localization was implemented using point resolved spectroscopy (PRESS) pulses (Bottomley, 1987). The dACC voxel was always acquired before left and right basal ganglia voxels in the same order. Water suppression was achieved with the help of chemical shift-selective (CHESS) pulses. Numerically optimized Shinnar-Le Roux radio frequency pulses were used for PRESS $\left(90^{\circ}, 180^{\circ}, 180^{\circ}\right)$ and CHESS $\left(90^{\circ}, 90^{\circ}, 90^{\circ}\right)$ (Haase et al, 1985). The parameters used for spectral acquisition were $\mathrm{TR}=3000 \mathrm{~ms}, \mathrm{TE}=30 \mathrm{~ms}$, sampling size $=1024,128$ averages. Four unsuppressed water free induction decay spectra were also acquired for eddy current suppression and phase correction
(Klose, 1990). The scan was acquired using a bandwidth of $2000 \mathrm{~Hz}$ and using 2048 complex data points. The FWHM of the unsuppressed water peak was optimized to $<14 \mathrm{~Hz}$.

MRS post-processing. The raw files were processed using LC Model package-Version 6-2-2b (Provencher, 2012). The LC Model is an operator-independent commercial software package that fits in vivo metabolite spectra using model resonances acquired under comparable scanning conditions from multiple compounds in standard phantom solutions. The water-suppressed time-domain data were analyzed between 1.0 p.p.m. and 4.0 p.p.m. without further $\mathrm{T} 1$ and $\mathrm{T} 2$ corrections. The basis set provided by the vendor of the LC Model (http://s-provencher.com/pages/lcm-applications. shtml) was used and then scaled to consistent receiver gain. It should be noted that the 6-2-2b version of LC Model when used with a short TE $(<30 \mathrm{~ms})$ sequence in high field strength (3T) can be scaled to provide relatively accurate estimates of glutamate (Glu). Nevertheless, as recommended by the vendor, concentrations and ratios with Cramer-Rao lower bounds (CRLB) $>20 \%$ were not used for analysis. The CRLB for glutamate (Glu) was reliably $<10 \%$, indicating low metabolite variance and good fit. Of note, glutamine resonances were fit poorly with CRLB ( $>20 \%$ ) and hence were not used in the analysis. Water was used as the internal reference, and creatine + phosphocreatine ( $\mathrm{Cr}$ ) was used to scale the data to model spectra across subjects (per LC Model Manual) and to minimize intra- and inter-individual variation. No significant differences in $\mathrm{Cr}$ concentrations were seen between IFN-alpha and control groups in any of the voxels at visit 1 or visit 2 , and no significant differences in $\mathrm{Cr}$ were seen between visit 1 and visit 2 for either group. All analyses used the $\mathrm{Glu} / \mathrm{Cr}$ ratio and were conducted by investigators blinded to group assignment.

\section{Statistical Analysis}

Student's $t$-tests and Fisher's exact tests, where appropriate, were used to compare groups in socio-demographic and
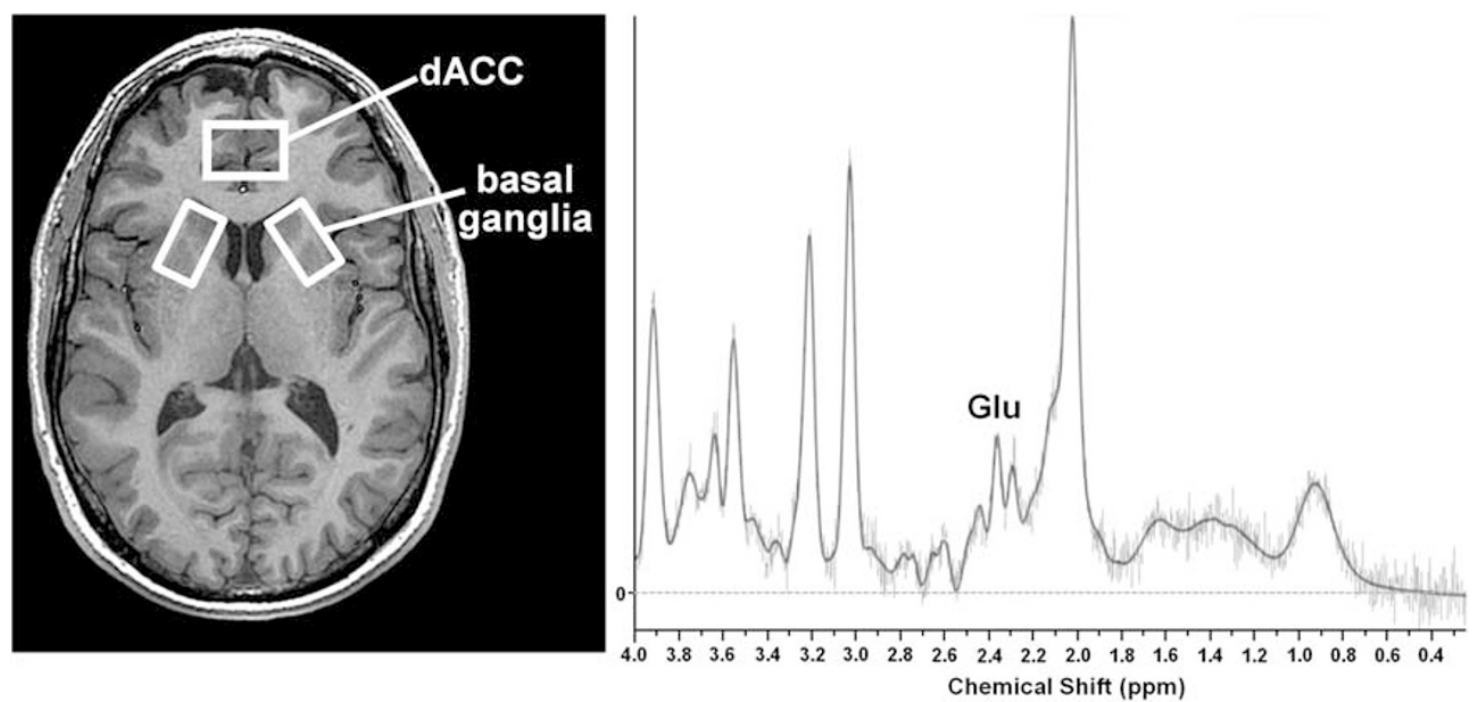

Figure I Placement of MRS voxels and representative spectrum: MRS voxels were placed as indicated: a $2 \times 3 \times 1-\mathrm{cm}^{3}$ voxel in the dorsal anterior cingulate region $(\mathrm{dACC})(\mathrm{BA} 24)$ and two $1.7 \times 3 \times 1.7-\mathrm{cm}^{3}$ voxels in the left and right basal ganglia. A representative spectrum from the $\mathrm{dACC}$ is depicted. 
clinical variables at baseline. To examine main effects of group and time and their interaction for relevant dependent variables (eg behavior, $\mathrm{Glu} / \mathrm{Cr}$, and cytokines), repeated measures analysis of variance was used. Bonferroni post hoc tests were used to compare specific means of interest. Delta values for the dependent measures were calculated by subtracting visit 1 from visit 2 and compared using Student's $t$-test. Delta values were also used to explore correlations between relevant dependent measures using Spearman coefficients for non-normally distributed data. Bonferroni correction was used to control for multiple comparisons where indicated. To control for relevant demographic and clinical covariates, partial correlation coefficients were employed. All statistical computations were performed using IBM-SPSS (International Business Machines, New York, NY). Of note, owing to poor scan quality, there was missing data for the following: dACC: one IFN-alpha-treated subject at visit 1; left basal ganglia: 2 IFNalpha-treated subjects (both visit 1); right basal ganglia: 1 control subject at visit 1 and 2 treatment patients (both visit 2). Subjects were excluded from analyses only for brain regions where imaging data was missing.

\section{RESULTS}

Of 46 subjects who signed informed consent, 31 (17 IFNalpha-treated patients and $14 \mathrm{HCV}$ controls) met entry criteria and were enrolled in the study. Excluded patients did not differ significantly from the study group on any of the socio-demographic or clinical variables (data not shown). No significant differences between IFN-alphatreated patients and controls were found with regard to age, sex, race, BMI, education, employment status, and history of substance abuse or depression, although there was a trend for a higher BMI $(p=0.06)$ and a numerically higher number of males $(p=0.14)$ in the IFN-alpha treatment group (Table 1).

\section{Behavioral Symptoms}

A significant main effect of time and a group-by-time interaction was found for depressive symptom severity as measured by the IDS-C $(\mathrm{F}(1,29)=27.03, p<0.001$ and $\mathrm{F}(1,29)=17.90, p<0.001$, respectively) with IFN-alpha- treated patients exhibiting significant increases in depression severity from visit 1 to visit 2 compared with controls (Table 2). Similar results were found for total MFI scores with a main effect of time and a significant group-by-time interaction $(\mathrm{F}(1,29)=5.68, p=0.024$ and $\mathrm{F}(1,29)=11.81$, $p<0.005$, respectively) (Table 2). Three of the five MFI subscales also exhibited a significant group-by-time interaction including general fatigue $(\mathrm{F}(1,29)=4.47, p=0.043)$, physical fatigue $(\mathrm{F}(1,29)=5.35, p=0.028)$, and reduced activity $(\mathrm{F}(1,29)=10.00, p<0.005)$ with significant increases in IFN-alpha-treated subjects from visit 1 to visit 2 compared with controls (Table 2). Of note, the three IFN-alpha-treated patients with a depression history showed no difference in depression scores during IFN-alpha compared with IFN-alpha-treated patients without a depression history $(p=0.60)$.

\section{Glu/Cr Ratio}

The baseline (visit 1) Glu/Cr ratio was not different between IFN-alpha-treated and HCV-control groups for the dACC (1.23 SD 0.19 vs 1.32 SD 0.16, respectively; $t=-1.28$, $\mathrm{df}=28, p=0.21)$, left basal ganglia (0.86 SD 0.17 vs $0.95 \mathrm{SD}$ $0.23 ; t=-1.14, \mathrm{df}=27, p=0.26$ ), or right basal ganglia (0.84 SD 0.20 vs 0.90 SD $0.20 ; t=-0.79, \mathrm{df}=28, p=0.43$ ). There were also no main effects of group or time for any of these brain regions. A significant group-by-time interaction was found for the Glu/Cr ratio in the dACC $(\mathrm{F}(1,28)=15.29$, $p<0.001)$ and left basal ganglia $(\mathrm{F}(1,27)=4.39, p=0.046)$ with IFN-alpha-treated patients exhibiting a significant increase in $\mathrm{Glu} / \mathrm{Cr}$ from visit 1 to visit 2 in both brain regions compared with controls (Figure 2). No main effects of group or time or group-by-time interaction were found for $\mathrm{Glu} / \mathrm{Cr}$ in the right basal ganglia.

\section{Inflammatory Cytokines}

As previously reported, there was a significant groupby-time interaction for plasma $\operatorname{TNF}(\mathrm{F}(1,29)=4.84$, $p=0.036)$ and a main effect of time and a group-bytime interaction for sTNFR2 $(\mathrm{F}(1,29)=18.59, p<0.001$ and $\mathrm{F}(1,29)=9.81, \quad p<0.005$, respectively) with significant increases in both inflammatory mediators in IFN-alphatreated patients from visit 1 to visit 2 compared with controls (Table 2).

Table I Sample Characteristics

\begin{tabular}{lccc}
\hline Variable & IFN-alpha $(\mathbf{n}=\mathbf{I 7})$ & HCV controls $(\mathbf{n}=\mathbf{I 4 )}$ & $\mathbf{p - v a l u e ~}$ \\
\hline Age mean years (SD) & $54.3(8.6)$ & $55.6(3.7)$ & $3(21 \%)$ \\
Sex no. females (\%) & $8(47 \%)$ & $6(43 \%)$ & 0.58 \\
Race no. Caucasian (\%) & $9(56 \%)$ & $13.8(3.5)$ & $10(71 \%)$ \\
Education years (SD) & $14.1(2.7)$ & $27.6(3.0)$ & 0.46 \\
Employed (yes or no) $n(\%)$ & $10(59 \%)$ & $4(29 \%)$ & 0.78 \\
Body mass index (SD) & $30.5(4.9)$ & $3(21 \%)$ & 0.75 \\
History of substance abuse & $5(29 \%)$ & 0.06 \\
History of depression & $3(18 \%)$ & 1.0 \\
\hline
\end{tabular}

Abbreviations: HCV, hepatitis C virus; IFN, interferon. 
Table 2 Behavioral and Inflammatory Measures Before (visit I) and During (visit 2) Interferon-Alpha

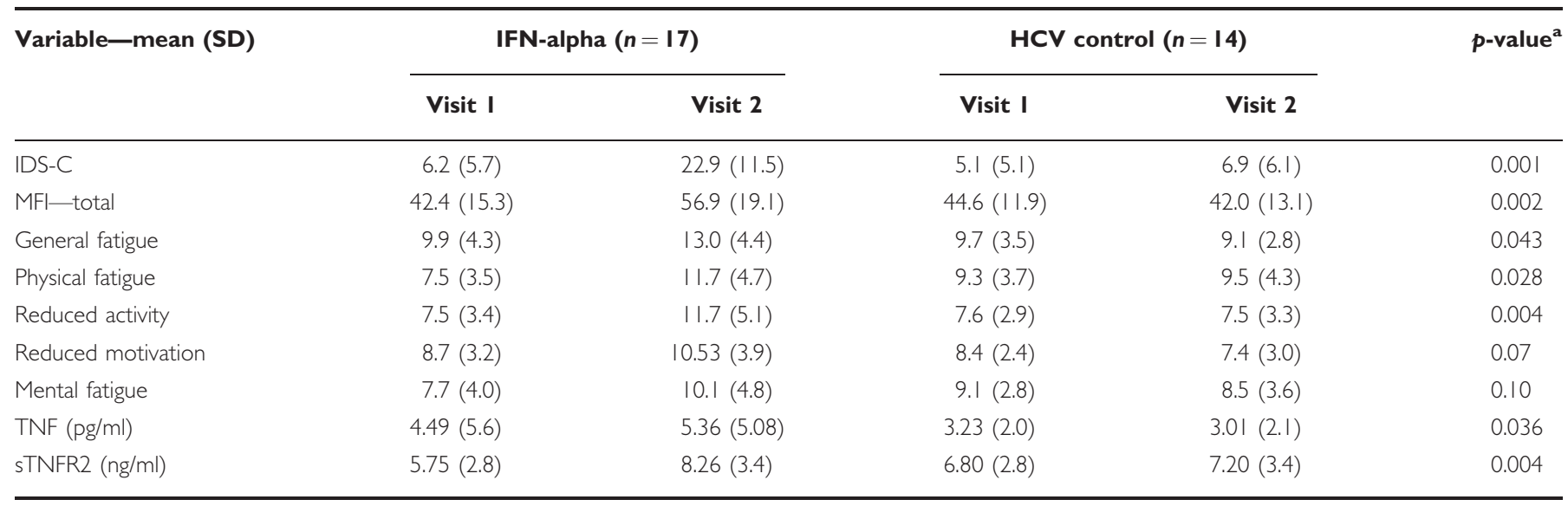

Abbreviations: HCV, hepatitis C virus; IDS-C, Inventory for Depressive Symptoms-Clinician Rating; IFN, interferon; MFI, Multidimensional Fatigue Inventory; TNF, tumor necrosis factor.

${ }^{a} p$-value is from the treatment $\times$ time interaction (repeated measures ANOVA interaction term).

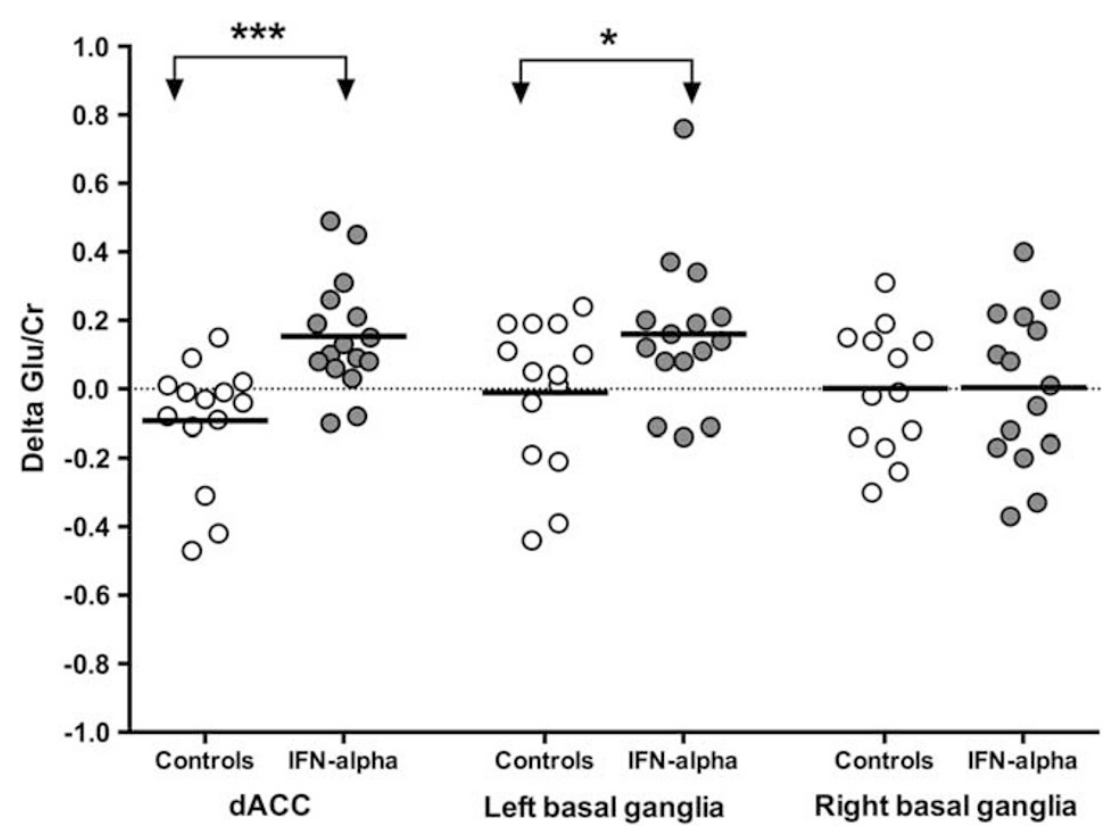

Figure 2 IFN-alpha increases Glu/Cr in dACC and left basal ganglia region. Regional changes in glutamate concentrations normalized to creatine (delta $\mathrm{Glu} / \mathrm{Cr}$ ) between visit I and 2 in the dorsal anterior cingulate cortex (dACC), left and right basal ganglia regions are depicted in interferon (IFN)-alphatreated (closed circles) and control subjects (open circles). Delta Glu/Cr (visit 2 - visit I) was significantly increased in IFN-alpha-treated patients compared with controls in the dACC and left basal ganglia $(t=3.91, \mathrm{df}=28, p<0.001$ and $t=2.10, \mathrm{df}=27, p=0.046$, respectively). No changes were seen in the right basal ganglia. $* p<0.05, * * * * 0.001$.

\section{Correlations}

$\mathrm{Glu} / \mathrm{Cr}$ and behavioral assessments. Correlations were assessed between delta $\mathrm{Glu} / \mathrm{Cr}$ in dACC and left basal ganglia and delta depression severity and fatigue correcting for multiple comparisons ( 2 brain regions $\times 6$ behavioral endpoints $=12$ comparisons). Delta $\mathrm{Glu} / \mathrm{Cr}$ in the dACC in the group as a whole was significantly correlated with delta depression severity (IDS-C) $\left(r_{\mathrm{s}}=0.54, \mathrm{df}=28, p=0.002\right.$, $\left.p_{\text {corr }}=0.024\right)$ but was not significantly correlated with delta fatigue subscale scores (Figure 3). Splitting the groups into IFN-alpha-treated and HCV controls revealed no significant correlations between delta $\mathrm{Glu} / \mathrm{Cr}$ and IDS-C in either group alone, suggesting a correlation largely driven by the differences in $\mathrm{Glu} / \mathrm{Cr}$ and IDS-C between groups. In contrast, delta $\mathrm{Glu} / \mathrm{Cr}$ in left basal ganglia in the group as a whole was significantly correlated with the delta MFI subscale for reduced motivation $\left(r_{\mathrm{s}}=0.60, \mathrm{df}=27, p=\right.$ $\left.0.001, p_{\text {corr }}=0.012\right)$. No other significant correlations for left basal ganglia and behavioral endpoints were found. Splitting the groups into IFN-alpha-treated subjects and $\mathrm{HCV}$ controls revealed that delta $\mathrm{Glu} / \mathrm{Cr}$ in left basal ganglia was significantly correlated with reduced motivation 


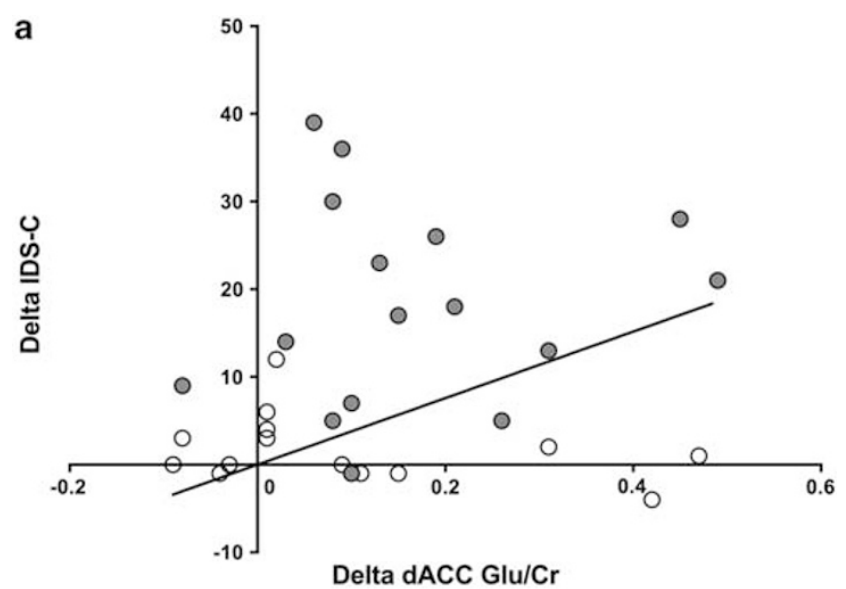

$p=0.018$ ), however, this relationship did not survive correction for multiple comparisons $\left(p_{\text {corr }}=0.072\right)$. No relationships were observed between delta $\mathrm{Glu} / \mathrm{Cr}$ in left basal ganglia and delta TNF markers.

\section{DISCUSSION}

The data presented herein demonstrate significant increases in glutamate as measured by the $\mathrm{Glu} / \mathrm{Cr}$ ratio using MRS in brain regions known to be targets of cytokine-induced behavioral changes including the dACC and left basal ganglia (Miller et al, 2013). Moreover, consistent with our previous work on the effects of IFN-alpha on neural activity in the ventral striatum and motivation (Capuron et al, 2012), increases in $\mathrm{Glu} / \mathrm{Cr}$ ratios in left basal ganglia significantly correlated with reduced motivation. Taken together, these data suggest that effects of inflammatory cytokines on the brain may be mediated in part by increased glutamate.

Under physiological conditions, the glutamate signal as measured by MRS is believed to be primarily derived from the intracellular compartment, which contains millimolar concentrations of glutamate as opposed to the extracellular compartment that contains concentrations in the nanomolar range, well below the detection limits of the MRS procedures employed in this and other studies (Mark et al, 2001; Potter et al, 2013). In part, related to its potential role in excitotoxicity, extracellular glutamate is maintained at these low concentrations by a family of excitatory amino-acid transporters (EAATs), which are located on the surface of astrocytes and oligodendrocytes and use a high-energy reuptake process to overcome the 1000-fold concentration gradient between intracellular and extracellular compartments (Schousboe and Waagepetersen, 2005; Tilleux and Hermans, 2007; Niciu et al, 2012). Under pathophysiologic conditions, however, glutamate concentrations in the extracellular space may significantly increase as a result of disruption in EAAT expression and/or function as well as alterations in glutamate metabolism. Relevant to the current findings, inflammatory cytokines including TNF have been shown in multiple studies to decrease the expression and function of EAATs. In addition, inflammatory cytokines can lead to reverse efflux of glutamate from astrocytes (Mark et al, 2001; Jourdain et al, 2007) and decrease the activity of glutamate metabolizing enzymes such as glutamine synthetase (Choudary et al, 2005). All of these changes can result in elevated extracellular glutamate concentrations as observed in this study with attendant consequences on neuronal integrity.

Consistent with the impact of inflammatory cytokines on glutamate metabolism, a number of brain disorders associated with neuroinflammation including brain trauma, multiple sclerosis, stroke, neurodegenerative disorders such as Huntington's disease, Alzheimer's disease, and infectious diseases such as HIV have been reported to be associated with increased glutamate as measured by MRS (Mark et al, 2001; Lee et al, 2003; Srinivasan et al, 2005; Mehta et al, 2013). Although it has been assumed that the increased glutamate in these disorders as well as those reported herein reflect an increase in the extracellular glutamate pool, 
without more definitive assessments, this conclusion cannot be verified (Mark et al, 2001).

An additional mechanism that may contribute to increased extracellular glutamate in IFN-alpha-treated patients is the kynurenine pathway. IFN-alpha as well as other inflammatory cytokines have been shown to stimulate the production of kynurenine that can be converted in the brain by microglia into quinolinic acid, which has been associated with IFN-alpha-induced depression (Walker et al, 2013). Quinolinic acid has been shown to stimulate synaptosomal glutamate release and block glutamate reuptake in astrocytes in vitro and stimulate glutamate release in rats in vivo (Tavares et al, 2002). Interestingly, increased quinolinic acid immunoreactive cells have been found in the same ACC region sampled in the current study (Steiner et al, 2011). Finally, ketamine, an antagonist at the NMDA receptor, has been shown to reverse depressive symptoms induced by the inflammatory stimulus, lipopolysaccharide, which has been shown to increase CNS quinolinic acid (Walker et al, 2013).

The data from IFN-alpha-treated patients are consistent with findings from patients with bipolar disorder where elevated glutamate concentrations in several ACC regions as well as the basal ganglia have been reported using MRS. Of relevance to inflammation, considerable data have documented increased peripheral blood inflammatory cytokines in bipolar patients as well as increased microglial activation and cytokine expression in the brain in postmortem samples. (Soczynska et al, 2009; Yuksel and Ongur, 2010; Leboyer et al, 2012). Unfortunately, however, correlations among glutamate concentrations, behavior, and inflammatory markers have not been conducted (or reported) in bipolar disorder.

The findings from this study contrast with recent results reporting no glutamate changes in a MRS voxel situated on the pregenual ACC in HCV patients undergoing IFN-alpha therapy (Taylor et al, 2013). Differences in study design involve our inclusion of a non-IFN-alpha-treated control group as well as our use of the traditional PRESS technique to assess glutamate concentrations as opposed to the 'SPECIAL' technique used to assess glutamate concentrations in the other study. Moreover, there can be considerable overlap between glutamate and glutamine signals, which may account for several glutamate/glutamine inconsistencies in the literature (Yuksel and Ongur, 2010). In a related fashion, the decreased rate of ${ }^{13} \mathrm{C}$ incorporation into the intracellular pool of glutamate and glutamine (reflected by decreased ${ }^{13} \mathrm{C}$ glutamate and ${ }^{13} \mathrm{C}$ glutamine as measured by MRS) following chronic unpredictable stress (CUS) in rats may represent cytokine-induced effects on glutamate reuptake and release leading to increased extracellular glutamate (Banasr et al, 2010). Indeed, behavioral effects of the CUS in mice have been shown to be mediated in part by inflammatory cytokines (Koo and Duman, 2008).

It should be mentioned that the mechanisms by which increased glutamate occurs in the basal ganglia and AACC may involve different processes. IFN-alpha has been associated with evidence of decreased dopamine similar to Parkinson's disease (PD) (Capuron et al, 2012). Thus, in addition to the direct effects of cytokines on glutamate metabolism, decreased availability of dopamine may indirectly lead to an overactivation of glutamatergic projections to the striatum and basal ganglia output nuclei as seen in PD (Hallett and Standaert, 2004; Paoletti et al, 2013). Indeed, D1 receptors in the direct pathway stimulate the release of GABA in the basal ganglia, whereas D2 receptors inhibit glutamate release in the indirect pathway. Depletion of dopamine would therefore be expected to decrease GABAergic inhibitory tone while at the same time increasing glutamatergic output. In contrast, the mechanism of glutamate increase in the dACC may be primarily related to direct effects of inflammatory cytokines on glutamate reuptake and release.

There are several strengths and weaknesses of the study that warrant consideration. We used a standard dose of an immune stimulus administered to medically stable patients off psychotropic medications without substance abuse and compared them with a control population. Limitations include the lack of randomization and blinding of behavioral ratings and the relatively short interval (1 month) between visits 1 and 2, which may have missed more chronic behavioral and neuroimaging changes. In addition, given the small sample size, there is insufficient data to conclusively determine the impact of hemispheric laterality on delta $\mathrm{Glu} / \mathrm{Cr}$ in the basal ganglia. There is a paucity of data on laterality of glutamate alterations in mood or inflammatory disorders (especially in basal ganglia). The relationship between laterality and depressive symptoms in patients with lesions of the basal ganglia has also been inconclusive, although several studies have supported a relationship between left basal ganglia lesions and depression following stroke and traumatic brain injury (Starkstein et al, 1988; Jorge et al, 1993; Paradiso et al, 2013). The absence of a non-HCV-infected control group as well as the combination of ribavirin, telaprevir, or boceprevir with IFN-alpha is also a limitation. However, previous work by our group and others has shown that IFNalpha effects on basal ganglia and dACC in HCV-infected patients mirror those seen in non-HCV-infected subjects treated with IFN-alpha alone including patients with cancer and non-human primates as well as healthy volunteers administered other inflammatory stimuli including typhoid vaccination and endotoxin (Miller et al, 2013).

\section{CONCLUSIONS}

This study provides support for the hypothesis that brain regions preferentially targeted by cytokines appear to show elevations in glutamate concentrations that correlate with IFN-alpha-induced behavioral changes notably reduced motivation. Future studies using glutamate antagonists may be relevant in patients with chronic exposure to inflammatory cytokines including patients with mood disorders with increased inflammatory biomarkers.

\section{FUNDING AND DISCLOSURE}

This work was funded in part by grants from the National Institute of Mental Health K23MH091254 (EH) and the National Association for Research on Schizophrenia and Affective Disorders (EH). All financial disclosures are listed for each author: Andrew H Miller has served as a consultant for Abbott Laboratories, AstraZeneca, GlaxoSmithKline, Lundbeck Research USA, F Hoffmann-La Roche Ltd., 
Schering-Plough Research Institute and Wyeth/Pfizer Inc. and has received research support from Centocor Inc., GlaxoSmithKline, and Schering-Plough Research Institute. Thaddeus Pace has received financial compensation for scientific advisory board services at Questcor Pharmaceuticals, Inc. Ebrahim Haroon, Bobbi Woolwine, Samir Parekh, James Spivey, Xiangchuan Chen and Xiaoping $\mathrm{Hu}$ declare no conflict of interest.

\section{ACKNOWLEDGEMENTS}

We acknowledge Sharon Sen for assistance with inflammatory biomarker assessments, Ryan Ford and Anjana Pillai for patient referrals, and support from the Wallace $\mathrm{H}$ Coulter Biomedical Information Technology Center for image analysis.

\section{REFERENCES}

Ajilore O, Haroon E, Darwin C, Binesh N, Thomas A, Kumaran S et al (2005). Measurement of brain betabolites in type 2 diabetes and depression using proton magnetic resonance spectroscopy. Neuropsychopharmacology 30: S94.

Ajilore O, Haroon E, Kumaran S, Darwin C, Binesh N, Mintz J et al (2007). Measurement of brain metabolites in patients with type 2 diabetes and major depression using proton magnetic resonance spectroscopy. Neuropsychopharmacology 32: 1224-1231.

Banasr M, Chowdhury GM, Terwilliger R, Newton SS, Duman RS, Behar KL et al (2010). Glial pathology in an animal model of depression: reversal of stress-induced cellular, metabolic and behavioral deficits by the glutamate-modulating drug riluzole. Mol Psychiatry 15: 501-511.

Bottomley PA (1987). Spatial localization in NMR spectroscopy in vivo. Ann N Y Acad Sci 508: 333-348.

Brydon L, Harrison NA, Walker C, Steptoe A, Critchley HD (2008). Peripheral inflammation is associated with altered substantia nigra activity and psychomotor slowing in humans. Biol Psychiatry 63: 1022-1029.

Capuron L, Pagnoni G, Demetrashvili M, Woolwine BJ, Nemeroff CB, Berns GS et al (2005). Anterior cingulate activation and error processing during interferon-alpha treatment. Biol Psychiatry 58: 190-196.

Capuron L, Pagnoni G, Drake DF, Woolwine BJ, Spivey JR, Crowe RJ et al (2012). Dopaminergic mechanisms of reduced basal ganglia responses to hedonic reward during interferon alfa administration. Arch Gen Psychiatry 69: 1044-1053.

Choudary PV, Molnar M, Evans SJ, Tomita H, Li JZ, Vawter MP et al (2005). Altered cortical glutamatergic and GABAergic signal transmission with glial involvement in depression. Proc Natl Acad Sci USA 102: 15653-15658.

Eisenberger NI, Berkman ET, Inagaki TK, Rameson LT, Mashal NM, Irwin MR (2010). Inflammation-induced anhedonia: endotoxin reduces ventral striatum responses to reward. Biol Psychiatry 68: 748-754.

First MB, Spitzer RL, Gibbon M, Williams JBW (2002). Structured Clinical Interview for DSM-IV-TR Axis I Disorders, Research Version, Patient Edition. (SCID-I/P). Biometrics Research, New York State Psychiatric Institute: New York.

Haase A, Frahm J, Hanicke W, Matthaei D (1985). 1H NMR chemical shift selective (CHESS) imaging. Phys Med Biol 30: 341-344.

Hallett PJ, Standaert DG (2004). Rationale for and use of NMDA receptor antagonists in Parkinson's disease. Pharmacol Ther 102: 155-174.

Haroon E, Watari K, Thomas A, Ajilore O, Mintz J, ElderkinThompson V et al (2009). Prefrontal myo-inositol concentration and visuospatial functioning among diabetic depressed patients. Psychiatry Res 171: 10-19.

Harrison NA, Brydon L, Walker C, Gray MA, Steptoe A, Dolan RJ et al (2009). Neural origins of human sickness in interoceptive responses to inflammation. Biol Psychiatry 66: 415-422.

Ida T, Hara M, Nakamura Y, Kozaki S, Tsunoda S, Ihara H (2008). Cytokine-induced enhancement of calcium-dependent glutamate release from astrocytes mediated by nitric oxide. Neurosci Lett 432: 232-236.

Jorge RE, Robinson RG, Arndt SV, Starkstein SE, Forrester AW, Geisler F (1993). Depression following traumatic brain injury: a 1 year longitudinal study. J Affect Disord 27: 233-243.

Jourdain P, Bergersen LH, Bhaukaurally K, Bezzi P, Santello M, Domercq $M$ et al (2007). Glutamate exocytosis from astrocytes controls synaptic strength. Nat Neurosci 10: 331-339.

Klose U (1990). In vivo proton spectroscopy in presence of eddy currents. Magn Reson Med 14: 26-30.

Koo JW, Duman RS (2008). IL-1beta is an essential mediator of the antineurogenic and anhedonic effects of stress. Proc Natl Acad Sci USA 105: 751-756.

Leboyer M, Soreca I, Scott J, Frye M, Henry C, Tamouza R et al (2012). Can bipolar disorder be viewed as a multi-system inflammatory disease? J Affect Disord 141: 1-10.

Lee PL, Yiannoutsos CT, Ernst T, Chang L, Marra CM, Jarvik JG et al Consortium tHM (2003). A multi-center1H MRS study of the AIDS dementia complex: Validation and preliminary analysis. J Magn Reson Imaging 17: 625-633.

Mark LP, Prost RW, Ulmer JL, Smith MM, Daniels DL, Strottmann JM et al (2001). Pictorial review of glutamate excitotoxicity: fundamental concepts for neuroimaging. AJNR Am J Neuroradiol 22: 1813-1824.

Mehta A, Prabhakar M, Kumar P, Deshmukh R, Sharma PL (2013). Excitotoxicity: bridge to various triggers in neurodegenerative disorders. Eur J Pharmacol 698: 6-18.

Miller AH, Haroon E, Raison CL, Felger JC (2013). Cytokine targets in the brain: impact on neurotransmitters and neurocircuits. Depress Anxiety 30: 297-306.

Niciu MJ, Kelmendi B, Sanacora G (2012). Overview of glutamatergic neurotransmission in the nervous system. Pharmacol Biochem Behav 100: 656-664.

Paoletti P, Bellone C, Zhou Q (2013). NMDA receptor subunit diversity: impact on receptor properties, synaptic plasticity and disease. Nat Rev Neurosci 14: 383-400.

Paradiso S, Ostedgaard K, Vaidya J, Ponto LB, Robinson R (2013). Emotional blunting following left basal ganglia stroke: the role of depression and fronto-limbic functional alterations. Psychiatry Res 211: 148-159.

Potter MC, Figuera-Losada M, Rojas C, Slusher BS (2013). Targeting the glutamatergic system for the treatment of HIV-associated neurocognitive disorders. J Neuroimmune Pharmacol 8: 594-607.

Provencher SK (2012). LCModel1 \& LCMgui User's Manual. Stephen Provencher 1-178.

Raison CL, Borisov AS, Majer M, Drake DF, Pagnoni G, Woolwine BJ et al (2009). Activation of central nervous system inflammatory pathways by interferon-alpha: relationship to monoamines and depression. Biol Psychiatry 65: 296-303.

Sanacora G, Treccani G, Popoli M (2012). Towards a glutamate hypothesis of depression: an emerging frontier of neuropsychopharmacology for mood disorders. Neuropharmacology 62: 63-77.

Sas AR, Bimonte-Nelson H, Smothers CT, Woodward J, Tyor WR (2009). Interferon-alpha causes neuronal dysfunction in encephalitis. J Neurosci 29: 3948-3955.

Schousboe A, Waagepetersen HS (2005). Role of astrocytes in glutamate homeostasis: implications for excitotoxicity. Neurotox Res 8: 221-225.

Slavich GM, Way BM, Eisenberger NI, Taylor SE (2010). Neural sensitivity to social rejection is associated with inflammatory 
responses to social stress. Proc Natl Acad Sci USA 107: 14817-14822.

Smets EM, Garssen B, Bonke B, De Haes JC (1995). The multidimensional fatigue inventory (MFI) psychometric qualities of an instrument to assess fatigue. J Psychosom Res 39: 315-325.

Soczynska JK, Kennedy SH, Goldstein BI, Lachowski A, Woldeyohannes HO, McIntyre RS (2009). The effect of tumor necrosis factor antagonists on mood and mental healthassociated quality of life: novel hypothesis-driven treatments for bipolar depression? Neurotoxicology 30: 497-521.

Srinivasan R, Sailasuta N, Hurd R, Nelson S, Pelletier D (2005). Evidence of elevated glutamate in multiple sclerosis using magnetic resonance spectroscopy at 3T. Brain 128: 1016-1025.

Starkstein SE, Robinson RG, Berthier ML, Parikh RM, Price TR (1988). Differential mood changes following basal ganglia vs thalamic lesions. Arch Neurol 45: 725-730.

Stein KD, Jacobsen PB, Blanchard CM, Thors C (2004). Further validation of the multidimensional fatigue symptom inventoryshort form. J Pain Symptom Manage 27: 14-23.

Steiner J, Walter M, Gos T, Guillemin GJ, Bernstein HG, Sarnyai $\mathrm{Z}$ et al (2011). Severe depression is associated with increased microglial quinolinic acid in subregions of the anterior cingulate gyrus: evidence for an immune-modulated glutamatergic neurotransmission? J Neuroinflammation 8: 94.

Stertz L, Magalhaes PV, Kapczinski F (2013). Is bipolar disorder an inflammatory condition? The relevance of microglial activation. Curr Opin Psychiatry 26: 19-26.
Tavares RG, Tasca CI, Santos CE, Alves LB, Porciuncula LO, Emanuelli T et al (2002). Quinolinic acid stimulates synaptosomal glutamate release and inhibits glutamate uptake into astrocytes. Neurochem Int 40: 621-627.

Taylor MJ, Godlewska B, Near J, Christmas D, Potokar J, Collier J et al (2013). Effect of interferon-alpha on cortical glutamate in patients with hepatitis C: a proton magnetic resonance spectroscopy study. Psychol Med 44: 789-795.

Tilleux S, Hermans E (2007). Neuroinflammation and regulation of glial glutamate uptake in neurological disorders. J Neurosci Res 85: 2059-2070.

Trivedi MH, Rush AJ, Ibrahim HM, Carmody TJ, Biggs MM, Suppes $\mathrm{T}$ et al (2004). The Inventory of Depressive Symptomatology, Clinician Rating (IDS-C) and Self-Report (IDS-SR), and the Quick Inventory of Depressive Symptomatology, Clinician Rating (QIDS-C) and Self-Report (QIDS-SR) in public sector patients with mood disorders: a psychometric evaluation. Psychol Med 34: $73-82$.

van der Graaf M (2010). In vivo magnetic resonance spectroscopy: basic methodology and clinical applications. Eur Biophys J 39: 527-540.

Walker AK, Budac DP, Bisulco S, Lee AW, Smith RA, Beenders B et al (2013). NMDA receptor blockade by ketamine abrogates lipopolysaccharide-induced depressive-like behavior in C57BL/ 6J mice. Neuropsychopharmacology 38: 1609-1616.

Yuksel C, Ongur D (2010). Magnetic resonance spectroscopy studies of glutamate-related abnormalities in mood disorders. Biol Psychiatry 68: 785-794. 